\title{
Atividade física e sintomas neuropsiquiátricos em pacientes com demência de Alzheimer
}

\author{
Salma Stéphany Soleman Hernandez ${ }^{1}$ \\ Thays Martins Vital $^{1}$ \\ Sebastião Gobbi ${ }^{1}$ \\ José Luiz Riani Costa ${ }^{1}$ \\ Florindo Stella ${ }^{1,2}$ \\ ${ }^{1}$ Instituto de Biociências. UNESP - Univ Estadual Paulista, Campus de Rio Claro, \\ Departamento de Educação Física, Laboratório de Atividade Física e Envelhecimento \\ (LAFE), Rio Claro, SP, Brasil \\ ${ }^{2}$ Ambulatório de Psiquiatria Geriátrica, Hospital das Clínicas, UNICAMP - Universidade \\ Estadual de Campinas, Campinas, SP, Brasil - Convênio CRUESP
}

\begin{abstract}
Resumo: Na demência de Alzheimer (DA) é comum a presença de sintomas neuropsiquiátricos resultantes de mudanças anatômicas e bioquímicas no cérebro. O objetivo deste estudo foi realizar uma revisão sistemática dos estudos que investigaram a relação entre atividade física e sintomas neuropsiquiátricos na DA. Efetuou-se uma pesquisa nas bases de dados: Academic Search Premier, Biological Abstracts, Medline, PsycINFO, Pubmed, Scielo, Science Direct, Sport Discus e Web of Science, utilizando-se as seguintes palavras - chave e operadores boleanos: (Neuropsychiatric disturbances OR Neuropsychiatric symptoms OR Neuropsychological disturbances OR Aberrant behavior OR Aberrant behaviour OR Behavior disturbances OR Behaviour disturbances OR Behavior management OR Behaviour management OR Disruptive behavior OR Disruptive behaviour) AND (Alzheimer) AND (Exercise OR Motor intervention OR training OR Physical activity OR fitness), além de referências cruzadas dos artigos selecionados. Foram encontrados oito estudos que preencheram os critérios de inclusão. Concluiu-se que bons resultados têm sido evidenciados para os distúrbios de agitação e sono de pacientes com DA. Entretanto então há um consenso de qual o melhor tipo de atividade para estes pacientes.
\end{abstract}

Palavras-chave: Exercício físico. Sintomas comportamentais. Doença de Alzheimer. Idoso.

\section{Physical activity and neuropsychiatric symptoms in elderly with Alzheimer's dementia}

\begin{abstract}
Is common the presence of neuropsychiatric symptoms in Alzheimer's disease (AD), this is resulted of anatomical and biochemical changes in the brain. The objective of present study was to look for papers relating physical activity and neuropsychiatric symptoms through a systematic review. The following data bases was accessed: Academic Search Premier, Biological Abstracts, Medline, PsycINFO, Pubmed, Scielo, Science direct on line, Sport Discus and Web of Science, using the following key-words: (Neuropsychiatric disturbances OR Neuropsychiatric symptoms OR Neuropsychological disturbances OR Aberrant behavior OR Aberrant behaviour OR Behavior disturbances OR Behaviour disturbances OR Behavior management OR Behaviour management OR Disruptive behavior OR Disruptive behaviour) AND (Alzheimer) AND (Exercise OR Motor intervention OR training OR Physical activity OR fitness), besides crossing the references in the selected articles. Eight studies had filled the inclusion criteria. It was possible to conclude there are evidences of positive results for agitation and sleep disturbances in patients with AD. However, there's no consensus of what kind of activity is better for these patients.
\end{abstract}

Key-words: Exercise. Behavioral symptoms. Alzheimer disease. Aged.

\section{Introdução}

A demência de Alzheimer (DA) é a uma doença neurodegenerativa caracterizada pelo acúmulo de placas amilóides extraneuronais e emaranhados neurofibrilares intraneuronais, principalmente, em regiões do lobo temporal, que determinam o declínio cognitivo progressivo (NITRINI et al., 2004).
Além das alterações cognitivas, a presença de sintomas neuropsiquiátricos é comum na DA e resultam de mudanças anatômicas e bioquímicas que ocorrem nesta patologia (LYKETSOS et al., 2001; CUMMINGS, 2003). Os sintomas neuropsiquiátricos geralmente compreendem domínios de comportamento, incluindo: delírios, alucinações, agitação, agressão, depressão, disforia, ansiedade, elação, euforia, apatia, indiferença, desinibição, irritação, labilidade, 
comportamento motor aberrante, alterações do sono e alterações de apetite e distúrbios alimentares (MEGA et al., 1996; CUMMINGS et al., 1994).

Tais sintomas apresentam uma freqüência relevante, acometendo entre 50 e $80 \%$ dos pacientes com DA (ASSAL; CUMMINGS, 2003), e concebem problemas clínicos importantes resultando em aumento do declínio funcional (HARWOOD et al. 2000), sobrecarga do cuidador (TAN et al. 2005), agressão (SASAKI et al. 2007), institucionalização (MAGNI et al. 1996; SCARMEAS et al. 2005) e aumento da mortalidade (TUN et al. 2007).

No Brasil, Tatsch et al., (2006) constataram que em 60 pacientes com DA, 78.3\% apresentavam um ou mais sintomas neuropsiquiátricos. Dentre eles, os mais freqüentes eram: apatia $(53.3 \%)$, depressão (38.3\%), alterações de sono (38.3\%) e ansiedade (25\%).

Ainda não há um tratamento definitivo que possa reverter a deterioração do funcionamento cognitivo e comportamental causada pela DA. Atualmente, o tratamento farmacológico mais empregado consiste na prescrição de anticolinesterásicos (rivastigmina, donepezil e galantamina) e de memantina (antiglutamatérgico), tanto para declínio cognitivo, quanto para distúrbios de comportamento (SEOW; GAUTHIER, 2007).

Uma grande variedade de intervenções não farmacológicas, como as intervenções sensoriais, ambientais, exercícios físicos e terapias comportamentais, têm sido evidenciadas como efetivas para os comportamentos inapropriados de idosos com demência (COHEN-MANSFIELD, 2001).

Alguns estudos apontam a prática de atividade física como uma abordagem não farmacológica de prevenção ou atenuação do declínio cognitivo, funcional e comportamental de idosos com DA (HEYN et al. 2004; CHRISTOFOLETTI et al. 2007; COELHO et al. 2009; HERNANDEZ et al. 2010; BUSSE et al. 2009). Embora os estudos evidenciem a atividade física como uma abordagem não farmacológica eficiente para os sintomas neuropsiquiátricos, ainda persistem algumas indagações em relação aos métodos utilizados pelos mesmos, como: qual o melhor tipo de exercício, número de participantes, presença de grupo controle e instrumentos de avaliação entre outras. Além disso, mecanismos neurobiológicos que poderiam explicitar melhor a relação causa e efeito da atividade física nos sintomas neuropsiquiátricos pouco são investigados.

Diante da prevalência dos sintomas neuropsiquiátricos na DA e da relação destes sintomas com outros problemas clínicos e também das indagações que ainda cercam os possíveis efeitos da atividade física e das controvérsias metodológicas, como tamanho amostral, amostra institucionalizada ou residente na comunidade, entre outras, torna-se importante analisar os estudos sobre a atividade física e sintomas neuropsiquiátricos em pacientes com DA. Deste modo, o objetivo do presente trabalho foi 0 de buscar e analisar artigos relacionados com os efeitos da atividade física nos distúrbios neuropsiquiátricos de idosos com Demência de Alzheimer, por meio de uma revisão sistemática.

\section{Métodos}

O delineamento metodológico do presente estudo consistiu em uma busca e análise de artigos que relacionassem os efeitos da atividade física nos distúrbios neuropsiquiátricos de pacientes com Demência de Alzheimer por meio de uma revisão sistemática, orientada pela busca bibliográfica nas bases de dados: Academic Search Premier, Biological Abstracts, Medline, PsycINFO, Pubmed, Scielo, Science direct on line, Sport Discus e Web of Science. As palavraschave e os operadores boleanos utilizados foram: (Neuropsychiatric disturbances $O R$ Neuropsychiatric symptoms $O R$ Neuropsychological disturbances $O R$ Aberrant behavior $O R$ Aberrant behaviour $O R$ Behavior disturbances $O R$ Behaviour disturbances $O R$ Behavior management $O R$ Behaviour management $O R$ Disruptive behavior $O R$ Disruptive behaviour) $A N D$ (Alzheimer) $A N D$ (Exercise $O R$ Motor intervention $O R$ training $O R$ Physical activity $O R$ fitness). Também foi realizada a busca manual em listas de referências dos artigos identificados. A busca foi realizada em maio de 2009 e a seleção dos artigos baseou-se no objetivo proposto pelo estudo e pelos critérios de inclusão descritos a seguir.

Critérios de inclusão: estudos longitudinais, randomizados ou não; amostras constituídas por indivíduos com diagnóstico provável de DA, segundo os critérios de órgãos internacionais CID (ORGANIZACÃ̃ MUNDIAL DA SAÚDE, 1978); 
NINCDS/ADRDA (MCKHANN et al. 1984), CERAD (MORRIS et al. 1989) e outros consensos internacionais; estudos em que a amostra era composta por pacientes com DA mas que não tinham outros tipos de demências associadas; estudos contendo sintomas neuropsiquiátricos como variáveis dependentes e atividade física como variável independente. Pesquisados no período de 1990 até junho de 2009.

Após a análise dos artigos, os mesmos foram excluídos pelo título (quando não se relacionavam com o tema proposto) e, quando necessário, pelo resumo e pelos métodos do trabalho.

\section{Resultados}

Com base nos critérios definidos, a busca bibliográfica resultou em 477 artigos. Em uma triagem inicial foram excluídos 403 artigos nos quais o título não estava relacionado com o tema proposto. Posteriormente, foram excluídos 66 artigos com base nos resumos que não preenchiam os critérios adotados pelo estudo.

Em grande maioria, os estudos encontrados através da busca bibliográfica trataram de investigações farmacológicas e não continham a atividade física com variável independente, além disso, poucos estudos evidenciaram os critérios para o diagnóstico de DA dos pacientes.

A partir dessas exclusões, foram selecionados oito artigos. Estes constituíram o grupo de publicações definitivamente considerado para a análise proposta.

A figura abaixo ilustra as etapas de seleção dos artigos.

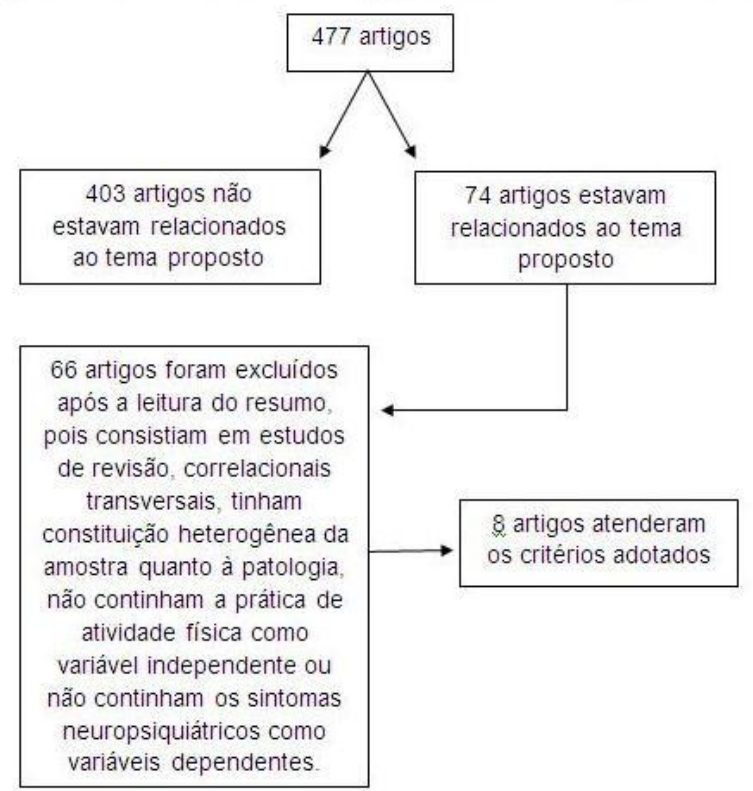

Figura 1. Etapas de seleção dos artigos incluídos na revisão sistemática
A seguir são descritos os artigos selecionados, por ano de publicação:

- Namazi et al., (1994) examinaram o efeito de um protocolo de atividade física no comportamento de 22 idosos institucionalizados. Os mesmos foram distribuídos igualmente no grupo intervenção e controle, com idade média de $80.7 \pm 5.8$ anos, $83.1 \pm 3.8$ anos, respectivamente. Para a avaliação do comportamento utilizou-se o Agitation Inventory Cohen-Mansfield e Billing (COHEN-MANSFIELD; BILLING, 1986).

O protocolo consistiu em treinamento combinado, 10 minutos de aquecimento e alongamento, seguidos de 30 minutos de exercícios que simulavam as atividades de vida diária, exercícios de flexibilidade, respiração e exercícios com pesos livres, por fim, 10 minutos de volta à calma.

Após 28 dias consecutivos de protocolo os autores concluíram que os participantes da atividade física reduziram pela metade os comportamentos de agitação.

- Namazi et al., (1995) abordaram 22 pacientes institucionalizados, destes, 11 pertenciam ao grupo intervenção e 11 ao grupo controle, com idade média de $80.7 \pm 5.8$ anos e 83.1 \pm 3.8 anos, respectivamente. Para avaliação da qualidade do sono, os autores propuseram um instrumento que mensura o comportamento do sono avaliado em períodos pré determinados.

O protocolo de atividade física foi do tipo combinado, pois continha mais de um tipo de exercício, como caminhada e exercícios resistidos com auxílio de halteres e caneleiras.

Após 336 horas de observação de sono, durante 28 dias consecutivos, os pacientes do grupo de intervenção motora apresentaram uma melhora na condição "dormindo" com um aumento de $25 \%$; além disso, obtiveram uma diminuição de $40 \%$ nos comportamentos agitados.

- $\quad$ Arkin et al., (1999) constataram que 11 idosos institucionalizados, com idade entre 59 e 89 anos, participantes de um programa de abordagem motora, cognitiva e social oferecido durante um ano, obtiveram uma melhora significativa na funcionalidade motora, cognição e socialização. Para avaliação dos sintomas depressivos foi utilizada a 30- item Geriatric Depression Scale (YESAVAGE et al. 1983). 
As sessões de exercícios foram dividas em aquecimento e parte principal, que por sua vez, foi composta por exercícios aeróbios e resistidos realizados em máquinas e volta à calma.

- Teri et al., (2003) compararam os comportamentos de 153 idosos, entre 55 e 93 anos, divididos, de forma randomizada, em um grupo que praticavam atividade física e um grupo que manteve a rotina médica/farmacológica, sem participar do programa.

Para mensurar os sintomas depressivos foi utilizada a escala de depressão Hamilton Depression Rating Scale (HAMILTON, 1960) e a escala Cornell Scale for Depression in Dementia (ALEXOPOULOS et al. 1988).

O programa aplicado foi composto por exercícios combinados (aeróbios, resistidos, equilíbrio e flexibilidade) e foram realizados na casa do participante, com uma duração de 30 minutos por sessão, com intensidade moderada.

Os autores concluíram que, após três meses da prática de atividade física, 68 participantes obtiveram melhoras nos sintomas depressivos. Após dois anos, os participantes ainda estavam mais preservados funcionalmente em comparação ao grupo de rotina e apresentavam menor institucionalização e menos distúrbios de comportamento.

- Heyn (2003) verificou os efeitos de um programa multisensorial por meio de estímulos físicos e cognitivos em 13 idosos institucionalizados, com idade média de $85.7 \pm 6.5$ anos.

Os comportamentos foram avaliados subjetivamente por meio do Menorah Park Engagement Scale (CAMP et al. 1995) e de um questionário desenvolvido pelo autor Caregiver Mood Report (ROLLAND et al. 2007).

O programa foi realizado três vezes por semana, durante oito semanas. Inicialmente, o tempo de execução das sessões foi de 15 minutos e gradualmente aumentado até completar 70 minutos.

Resultados benéficos foram encontrados no momento final do estudo em relação aos sintomas comportamentais dos participantes.

- Rolland et al., (2007) investigaram a participação de 134 idosos institucionalizados, com idade média de $83 \pm 7.4$ anos, em um protocolo de atividade física.
O Neuropsychiatric Inventory (CUMMINGS et al. 1994) e Montgomery and Asberg Depression Rating Scale (MULLER-THOMSEN et al. 2005) foram utilizados como instrumentos de avaliação.

A abordagem motora foi realizada duas vezes por semana, em dias não consecutivos, totalizando 88 sessões, e consistiu em exercícios aeróbios, de força, equilíbrio e flexibilidade.

Os autores não evidenciaram diferenças significativas para as variáveis relacionadas com os sintomas neuropsiquiátricos.

- $\quad$ Steinberg et al., (2008) adotaram o Neuropsychiatric Inventory (CUMMINGS et al. 1994) e a Cornell Scale for Depression in Dementia (ALEXOPOULOS et al. 1988) para avaliação dos sintomas neuropsiquiátricos.

Participaram deste estudo 27 sujeitos que foram randomizados em grupo controle e grupo intervenção com idade média, respectivamente, de $74 \pm 8.1$ anos e $76.5 \pm 3.9$ anos.

Após 12 semanas de treinamento, os dois grupos demonstraram uma tendência ao agravamento da depressão e qualidade de vida, e, portanto, sem diferença significativa entre os grupos para as variáveis neuropsiquiátricas. $O$ protocolo de atividade física consistiu em caminhada, exercícios resistidos com caneleiras e faixas elásticas, além de treinamento para equilíbrio e flexibilidade.

- $\quad$ Aman e Thomas (2009) verificaram uma redução no comportamento de agitação por meio da Pittisburgh Agitation Scale (ROSEN et al. 1991), após três semanas de treinamento combinado em 50 idosos institucionalizados.

O estudo ocorreu três vezes por semana, com duração de 30 minutos, durante três semanas. Os participantes tinham uma média de idade de 79.2 \pm 9.7 anos.

A depressão e agitação foram mensuradas por meio das escalas: Cornell Scale for Depression (ALEXOPOULOS et al. 1988) e Pittisburgh Agitation Scale/Cohen-Mansfield Agitation Inventory (ROSEN et al. 1991; MILLER et al. 1995), respectivamente.

As tabelas abaixo resumem as características metodológicas e resultados encontrados nos estudos citados anteriormente. 
Tabela1. Resumo das características metodológicas e resultados encontrados pelos estudos citados acima.

\begin{tabular}{|c|c|c|c|c|c|c|c|}
\hline Autor & $\begin{array}{l}\text { Tipo de } \\
\text { estudo }\end{array}$ & Ano & Sujeitos & Idade & Métodos & Intervenção & Resultados \\
\hline $\begin{array}{l}\text { Namazi } \\
\text { et al. }\end{array}$ & $\begin{array}{l}\text { Conveniência } \\
\text { Controlado }\end{array}$ & 1994 & $\begin{array}{c}22 \text { indivíduos } \\
\text { institucionalizados }\end{array}$ & $\begin{array}{c}\mathrm{GR}=83.1 \pm 3.8 \\
\mathrm{Gl}=80.7 \pm 5.8\end{array}$ & $\begin{array}{c}\text { Cohen-Mansfield } \\
\text { Agitation } \\
\text { Inventory; }\end{array}$ & $\begin{array}{l}\text { Treinamento } \\
\text { combinado } \\
28 \text { dias } \\
\text { consecutivos } \\
40 \text { ' /dia } \\
\end{array}$ & $\begin{array}{c}\text { Redução pela metade } \\
\text { nos comportamentos } \\
\text { agitados }\end{array}$ \\
\hline $\begin{array}{l}\text { Namazi } \\
\text { et al. }\end{array}$ & $\begin{array}{l}\text { Conveniência } \\
\text { Controlado }\end{array}$ & 1995 & $\begin{array}{c}22 \text { indivíduos } \\
\text { institucionalizados }\end{array}$ & $\begin{array}{c}\mathrm{GR}=83.1 \pm 3.8 \\
\mathrm{Gl}=80.7 \pm 5.8\end{array}$ & $\begin{array}{c}\text { Sleep behavior log } \\
\text { by time }\end{array}$ & $\begin{array}{c}\text { Treinamento } \\
\text { combinado } \\
28 \text { dias } \\
\text { consecutivos } \\
40 \text { ' /dia } \\
\end{array}$ & $\begin{array}{l}\text { Redução dos } \\
\text { comportamentos de } \\
\text { agitação no sono }\end{array}$ \\
\hline $\begin{array}{l}\text { Arkin } \\
\text { et al. }\end{array}$ & $\begin{array}{l}\text { Conveniência } \\
\text { Aberto }\end{array}$ & 1999 & $\begin{array}{c}14 \text { indivíduos } \\
\text { Institucionalizados }\end{array}$ & $59-89$ & $\begin{array}{l}\text { MEEM; Geriatric } \\
\text { Depression Scale; }\end{array}$ & $\begin{array}{l}\text { Treinamento } \\
\text { combinado } \\
48 \text { semanas - } 2 \\
\text { x semana } \\
\end{array}$ & $\begin{array}{l}\text { Melhoras significativas } \\
\text { nos sintomas depressivos }\end{array}$ \\
\hline $\begin{array}{l}\text { Teri } \\
\text { et al. }\end{array}$ & $\begin{array}{l}\text { Randomizado } \\
\text { Controlado }\end{array}$ & 2003 & $\begin{array}{l}153 \text { indivíduos } \\
\text { residentes na } \\
\text { comunidade }\end{array}$ & $55-93$ & $\begin{array}{c}\text { MEEM; } \\
\text { Hamilton } \\
\text { Depression Rating } \\
\text { Scale; } \\
\text { Cornell Scale for } \\
\text { Depression in } \\
\text { Dementia } \\
\end{array}$ & $\begin{array}{l}\text { Treinamento } \\
\text { combinado } \\
12 \text { semanas - } \\
30^{\prime} \text { por sessão }\end{array}$ & $\begin{array}{l}\text { Melhoras nos sintomas } \\
\text { depressivos }\end{array}$ \\
\hline Heyn & $\begin{array}{l}\text { Conveniência } \\
\text { Aberto }\end{array}$ & 2003 & $\begin{array}{c}13 \text { indivíduos } \\
\text { institucionalizados }\end{array}$ & $\begin{array}{c}70-93 \\
\text { (média }=85.7 \pm 6.5 \text { ) }\end{array}$ & $\begin{array}{c}\text { MEEM; Brief } \\
\text { Cognitive Rating } \\
\text { Scale; Menorah } \\
\text { Park Engagement } \\
\text { Scale; Caregiver } \\
\text { Mood Report; }\end{array}$ & $\begin{array}{l}\text { Treinamento } \\
\text { combinado } \\
8 \text { semanas }-3 x \\
\text { semana }-70^{\prime}\end{array}$ & $\begin{array}{c}\text { Benefícios nos sintomas } \\
\text { comportamentais e } \\
\text { cognitivos }\end{array}$ \\
\hline $\begin{array}{l}\text { Rolland } \\
\text { et al. }\end{array}$ & $\begin{array}{l}\text { Randomizado } \\
\text { Controlado }\end{array}$ & 2007 & $\begin{array}{l}134 \text { indivíduos } \\
\text { institucionalizados }\end{array}$ & $\begin{array}{c}62-103 \text { (média }= \\
83 \pm 7.4)\end{array}$ & $\begin{array}{c}\text { MEEM; } \\
\text { Neuropsychiatric } \\
\text { Inventory; } \\
\text { Montgomery and } \\
\text { Asberg Depression } \\
\text { Rating Scale; } \\
\end{array}$ & $\begin{array}{l}\text { Treinamento } \\
\text { combinado } \\
48 \text { semanas - } 2 \\
\text { x semana }\end{array}$ & $\begin{array}{c}\text { Não encontrou diferenças } \\
\text { significativas para os } \\
\text { distúrbios analisados }\end{array}$ \\
\hline $\begin{array}{l}\text { Steinberg } \\
\text { et al. }\end{array}$ & $\begin{array}{l}\text { Randomizado } \\
\text { Controlado }\end{array}$ & 2008 & $\begin{array}{l}27 \text { indivíduos } \\
\text { residentes } \\
\text { comunidade }\end{array}$ & $\begin{array}{l}\mathrm{GR}=74 \pm 8.1 \\
\mathrm{Gl}=76.5 \pm 3.9\end{array}$ & $\begin{array}{l}\text { A 1-h cognitive } \\
\text { battery; } \\
\text { Neuropsychiatric } \\
\text { Inventory; Cornell } \\
\text { Scale for } \\
\text { Depression in } \\
\text { Dementia; }\end{array}$ & $\begin{array}{l}\text { Treinamento } \\
\text { combinado } \\
12 \text { semanas }\end{array}$ & $\begin{array}{c}\text { Não encontrou diferenças } \\
\text { significativas para os } \\
\text { distúrbios analisados }\end{array}$ \\
\hline $\begin{array}{l}\text { Aman e } \\
\text { Thomas }\end{array}$ & $\begin{array}{l}\text { Conveniência } \\
\text { Controlado }\end{array}$ & 2009 & $\begin{array}{c}50 \text { indivíduos } \\
\text { institucionalizados }\end{array}$ & $78.8 \pm 8.7$ & $\begin{array}{l}\text { Cornell Scale for } \\
\text { Depression; } \\
\text { Pittisburgh } \\
\text { Agitation } \\
\text { Scale/Cohen- } \\
\text { Mansfield Agitation } \\
\text { Inventory; } \\
\end{array}$ & $\begin{array}{c}\text { Treinamento } \\
\text { combinado } \\
3 \text { semanas - } 3 x \\
\text { na semana - } \\
30^{\prime}\end{array}$ & $\begin{array}{c}\text { Redução no } \\
\text { comportamento de } \\
\text { agitação, contudo sem } \\
\text { diferenças para os } \\
\text { sintomas depressivos }\end{array}$ \\
\hline
\end{tabular}

\section{Discussão}

Após analisar os artigos selecionados, oito artigos foram incluídos no estudo definitivamente. Destes oito, seis estudos encontraram efeitos benéficos da atividade física nos sintomas neuropsiquiátricos avaliados.

Os distúrbios neuropsiquiátricos mais pesquisados pelos estudos encontrados nesta revisão foram, os sintomas depressivos, agitação e sono. Tendo em vista que os distúrbios neuropsiquiátricos na DA compreendem pelo menos dezoito categorias de sintomas e sinais diferentes (CUMMINGS et al. 1994; CUMMINGS, 2003), é possível evidenciar uma lacuna de estudos que abordem os outros tipos de distúrbios neuropsiquiátricos presentes nesta patologia.
De maneira a facilitar a análise e discussão dos estudos analisados foi feita uma divisão por distúrbio analisado em cada estudo.

\section{Sintomas depressivos}

O estudo de Arkin et al., (1999) verificou que um protocolo de atividade física sistematizado, por meio de um treinamento com pesos trouxe benefícios significativos para a diminuição dos sintomas depressivos de pacientes institucionalizados. Os autores descrevem uma boa sistematização do treinamento como a sobrecarga e individualidade na prescrição dos exercícios.

No entanto, lembram que ainda não está claro se os benefícios cognitivos, comportamentais e sociais podem ser relacionados com a atividade 
física por si ou quando isto também é efeito da interação social regular com outros indivíduos.

Teri et al., (2003) vão ao encontro do estudo citado anteriormente, pois também encontraram resultados benéficos da atividade física nos sintomas depressivos; contudo, em pacientes residentes na comunidade que realizavam as atividades em suas respectivas casas. Tal fato pode não garantir a intensidade, freqüência $e$ eficiência dos exercícios prescritos; em contrapartida, exclui o efeito da interação social questionada por Arkin et al., (1999).

Aspectos sociais, de interação com outros pacientes ou comunidade, estão cada vez mais fortemente explícitos na literatura, como nos estudos de Vance et al., (2002) e Bottino et al., (2002). Tais aspectos são relevantes e não deveriam ser vistos como fator limitante nos estudos e sim como uma característica intrínseca da prática da atividade física.

Rolland et al., (2007), Steinberg et al., (2008) e Aman e Thomas (2009) concluem que a atividade física não foi efetiva para surtir resultados benéficos nos sintomas depressivos de pacientes com DA.

Rolland et al., (2007) sugerem abordagens mais centradas apenas nos sintomas neuropsiquiátricos; também justificam o número de sessões, duas por semana ter sido insuficiente para surtir resultados significativos; no entanto, alertam para a possível redução da aderência dos pacientes caso sejam aumentadas as sessões semanais.

Steinberg et al., (2008) justificam que a pequena amostra (27 indivíduos) e natureza piloto do estudo limitam os resultados obtidos pelo estudo.

Aman e Thomas, (2009) obtiveram um grupo intervenção de 40 indivíduos versus 10 no grupo controle, colocando em risco as comparações realizadas e os resultados obtidos no estudo.

Os estudos citados anteriormente contêm características metodológicas bastante diferentes entre si e limitam conclusões mais fechadas acerca dos efeitos da prática da atividade física. Há uma importante variação do tempo de intervenção adotado: de 3 a 48 semanas e também em relação à randomização, presença de grupo controle e tamanho da amostral.
Arkin et al., (1999) constituíram uma amostra por conveniência, sem grupo controle, com 14 indivíduos, já Teri et al., (2003), Rolland et al., (2007) e Steinberg et al., (2008) constituíram uma amostra randomizada e controlada com tamanho amostral igual a 153, 134 e 27 indivíduos, respectivamente, Aman e Thomas (2009) uma amostra por conveniência, controlada com 50 participantes.

Outra característica em relação aos estudos analisados é a prevalência de amostras institucionalizadas que, de maneira geral, estão associadas a um menor desempenho funcional e cognitivo que poderão interferir diretamente nos resultados esperados pela prática de atividade física (PLATI et al. 2006).

Os estudos de Teri et al., (2003) e Steinberg et al., (2008) foram constituídos por indivíduos residentes na comunidade, entretanto, encontraram resultados contraditórios. Assim, mais estudos com amostras residentes na comunidade deverão ser realizados a fim de não somente responder se há efeito da prática da atividade física, mas também a fim de retardar a institucionalização precoce, o declínio funcional e cognitivo de pacientes com DA.

Dos cinco estudos discutidos acima, apenas dois apresentaram resultados benéficos em relação à prática de atividade física nos sintomas depressivos. De um modo geral, valorizam a necessidade de mais estudos para se chegar a um consenso, além de enriquecer as abordagens não farmacológicas como forma de tratamento para um dos distúrbios mais prevalentes na DA.

\section{Distúrbios de Agitação}

O conceito de agitação é complexo: às vezes, é tido como um distúrbio neuropsiquiátrico (TERI et al. 1988), outras vezes é dado como uma categoria que pode abranger outros comportamentos (COHEN-MANSFIELD; BILLING, 1986; COHEN-MANSFIELD, 1988).

São necessários mais estudos que compreendam esta problemática, a fim de não somente chegar a um consenso, mas também de encontrar respostas satisfatórias para este comportamento que pode afetar até $93 \%$ dos idosos institucionalizados (COHEN-MANSFIELD; BILLING, 1986).

Namazi et al., (1994) relatam que houve uma redução significativa (50\%) dos comportamentos 
de agitação após os pacientes terem participado por 28 dias consecutivos de um protocolo de atividade física onde as atividades de vida diária eram estimuladas. Os autores deste estudo observaram que os resultados não podem ser generalizados devido à pequena amostra constituinte (22 indivíduos) e o fato de os grupos não terem sido randomizados.

Aman e Thomas (2009) também fazem uma descrição detalhada do protocolo utilizado evidenciando 0 controle metodológico em variáveis como sobrecarga e individualidade. Concluíram que o grupo que participou da atividade oferecida foi significativamente melhor em comparação àqueles que compuseram 0 grupo controle. Entretanto, os grupos participantes eram diferentes no momento inicial da pesquisa em relação aos distúrbios de agitação, o que pode colocar em dúvida os resultados obtidos: o grupo treinamento apresentava significativamente menos comportamentos agitados quando comparado com o grupo controle.

Heyn (2003) verificou que um programa multisensorial pode ter bons resultados em pacientes com DA. Em seu estudo, a abordagem motora foi inserida juntamente com a estimulação cognitiva, através de exercícios de tarefa dupla. A própria autora alerta para o fato de o estudo ser limitado em relação à falta do grupo controle, a pequena amostra (13 indivíduos) e a não randomização da mesma.

Os três estudos apresentados fornecem aspectos positivos da prática de atividade física no distúrbio de agitação de pacientes com DA. Ambos têm características metodológicas semelhantes: amostras institucionalizadas, selecionadas por conveniência e pequeno tempo de intervenção: de quatro a oito semanas.

Ainda que a literatura venha evidenciando um efeito benéfico da prática da atividade física, pouco se discute a respeito de quais mecanismos estão envolvidos para a produção desta melhora.

Após a análise dos artigos descritos acima, é possível inferir que os distúrbios de agitação, em pacientes com DA, podem ser atenuados por meio da prática da atividade física. Contudo, a produção de novos estudos poderá contribuir para a criação de um consenso a respeito desta problemática, além disso, reforça-se a necessidade de estudos controlados, com amostras residentes na comunidade $e$ randomização da mesma.

\section{Distúrbios de Sono}

Distúrbios de sono são comuns em pacientes com DA. Estudos de caráter transversal com amostras tanto em instituições de longa permanência quanto na comunidade citam que $19-44 \%$ dos pacientes tem distúrbios de sono (RITCHIE, 1996; MCCURRY et al. 1999; CARPENTER et al. 1995).

Para McCurry et al., (2000) os distúrbios de sono são multifatoriais e são influenciados por uma variedade de fatores como demográficos, físicos e psiquiátricos, o que dificulta o tratamento e diagnóstico deste distúrbio.

Um único estudo sobre o efeito da atividade física no distúrbio de sono foi incluído nesta revisão (Namazi et al. 1995) que concluiu que a atividade física foi suficiente para reduzir a agitação no distúrbio de sono, aumentar a condição "dormindo" e "acordado mas, calmo", dos pacientes institucionalizados em relação ao grupo que não participou da atividade física.

Este estudo conta com um grupo controle que, além de aumentar a confiabilidade dos resultados encontrados, também exclui os efeitos da interação social, pois realizava atividades de leitura e poesia permitindo assim a interação social àqueles que não participavam da atividade física.

O estagiamento da doença deve ser uma ferramenta importante a ser controlada, a fim de não obter resultados negativos ou oscilatórios; com o avanço da doença é notório que os distúrbios muitas vezes se acentuam, ficando cada vez mais difícil o seu controle (TERI et al. 1988).

Os autores concluem que 0 fato de os pacientes estarem nos estágios leve e moderado da doença permitiu um melhor aproveitamento da atividade oferecida e, assim, resultou positivamente diante daqueles que ao estarem em estágios mais avançados, por vezes, nem participariam das atividades.

Este estudo vai ao encontro de outros que também verificaram benefícios da prática de atividade física no distúrbio de sono (MCCURRY et al. 2004; MCCURRY et al.2005).

Um aspecto importante a ser destacado em estudos com indivíduos com DA é o fato da 
percepção do paciente não corresponder com seu estado geral funcional e comportamental. De maneira geral, estes pacientes tendem a superestimar suas capacidades, pois simplesmente não compreendem seu estado de doença que muitas vezes é mascarado pela própria família (ALMEIDA; CROCCO, 2000).

Onor et al., (2006) confirmaram estes fatos quando submeteram grupos de pessoas com declínio cognitivo leve e DA a testes de cognição, distúrbios neuropsiquiátricos, atividades de vida diária, escala de percepção clínica e encontraram que ambos os grupos subestimaram déficits que eram considerados graves pelos cuidadores.

Estes fatos podem representar uma limitação, uma vez que a maioria dos estudos conta com testes onde o próprio paciente responde as perguntas. No estudo de Namazi et al., (1995) o questionário sobre o sono foi preenchido por um profissional da instituição e, desta forma, evitouse respostas super ou subestimadas por parte dos pacientes.

É evidente a demanda de mais estudos e o desenvolvimento de testes cada vez mais específicos em relação a esta população, de forma que as respostas sejam fidedignas aos comportamentos e não somente em relação à percepção do paciente.

O estudo discutido anteriormente mostrou efeitos positivos a respeito da influência da atividade física no distúrbio de sono de pacientes com DA. Contudo, ainda há carência de evidências que suportem este achado. Além disso, estudos que compreendam não somente os idosos institucionalizados como aqueles residentes na comunidade poderão mediar suporte para uma abordagem adequada.

\section{Conclusão}

Após analisar e discutir os estudos encontrados nesta revisão é possível inferir que ainda não há consenso a respeito da atividade física na redução nos sintomas depressivos de pacientes com DA. No entanto, bons resultados têm sido evidenciados para os distúrbios de agitação e sono dos mesmos.

Ainda não há indícios de qual o melhor tipo de atividade a ser conduzida para a melhora ou atenuação dos distúrbios neuropsiquiátricos de pacientes com DA. Por tal motivo, a atividade física ainda constitui uma problemática importante a ser investigada. Estudos que investiguem qual o melhor tipo, intensidade, freqüência e duração de exercícios que irão compor a atividade a ser desenvolvida poderão mediar uma melhora dos distúrbios neuropsiquiátricos destes pacientes.

Além disso, os componentes neurobiológicos envolvidos na prática da atividade física não foram investigados, evidenciando uma linha de estudo inovadora a ser pesquisada.

Portanto, a produção de mais estudos que constatem o efeito da prática da atividade física, que investiguem a especificidade dos exercícios, bem como os mecanismos de ação dos mesmos é necessária para o tratamento eficaz dos distúrbios neuropsiquiátricos de pacientes com DA.

\section{Referências}

ALEXOPOULOS, G. S.; ABRAMS, R. C.; YONG, R. C.; SHAMOIAN, C. A. Cornell scale for depression in dementia. Biological Psychiatry, Dallas, v. 23, p. 271-284, 1988.

ALMEIDA, O. P.; CROCCO, E. I. Perception of cognitive déficits and behavior disorders in patients with Alzheimer's disease. Arquivos de Neuropsiquiatria, São Paulo, v. 58, p. 292-299, 2000.

AMAN, E.; THOMAS, D. R. Supervised Exercise to Reduce Agitation in Severely Cognitively Impaired Persons. Journal of American Medical Directors Association, Atlanta, v. 10, p. 271-276, 2009.

ARKIN, S. M.; HOWELL, N. M. Elder Rehab: A Student-Supervised Exercise Program for Alzheimer's Patients. The Gerontologist, Cary, v. 39, n. 6, p. 279-775, 1999.

ASSAL, F.; CUMMINGS, J. L. Neuropsychiatric symptoms in the dementias. Current Opinion in Neurology, London, v. 15, n. 4, p. 445-450, 2003.

BOTTINO, M. C.; CARVALHO, I. A. M.; ALVAREZ, A. M. M. A.; ÁVLIA, R.; ZUKAUSKAS, S. E. Z.; ANDRADE, F. C.; HOTOTIAN, S. R.; S. A. F. F. I. F.; CAMARGO, C. H. P. Cognitive rehabilitation in Alzheimer's disease patients: multidisciplinary team report. Arquivos de Neuropsiquiatria, São Paulo, v. 60, n. 1, p. 7079, 2002.

BUSSE, A. L.; GIL, G.; SANTARÉM, J. M.; FILHO, W. J. Physical activity and cognition in the elderly. Dementia e Neuropsychologia, São Paulo, v. 3, n. 3, p. 204-208, 2009.

CAMP, C. J.; FOSS, J. W.; O'HANLON, A. M.; STEVENS, A. B. Memory interventions for persons with dementia. Applied Cognitive Psychology, Malden, v. 24, n. 4, p. 629-636, 1995. 
CARPENTER, B. D.; STRAUSS, M. E.; PATTERSON, M. B. Sleep disturbances in community-dwelling patients with Alzheimer's disease. Clinical Gerontologist, London, v. 16, p. 35-49, 1995.

CHRISTOFOLETTI, G.; OLIAN, I. M. M.; GOBBI, S.; STELLA, F. Effects of Motor Intervention in elderly patients with dementia: an analysis of randomized controlled trials. Topics in Geriatric Rehabilitation, Philadelphia, v. 23, n. 2, p. 14954, 2007.

COELHO, F. G. M.; GALDUROZ-SANTOS, R. F.; GOBBI, S.; STELLA, F. Atividade física sistematizada e desempenho cognitivo com demência de Alzheimer: uma revisão sistemática. Revista Brasileira de Psiquiatria, São Paulo, v. 31, p. 163-170, 2009.

COHEN-MANSFIELD, J.; BILLING, N. Agitated behaviors in the elderly: II. Journal of the American Geriatrics_Society, Los Angeles, v. 34, p. $711-727,1986$.

COHEN-MANSFIELD, J. Agitated behavior and cognitive functioning in nursing home residents: Preliminary results. Clinical Gerontologist, London, v. 7, p. 11-22, 1988.

COHEN-MANSFIELD, J. Nonpharmacologic interventions for inappropriate behaviors in dementia: a review, summary, and critique. American Journal of Geriatric Psychiatry, Bethesda, v. 9, p. 361-381, 2001.

CUMMINGS, J. L.; MEGA, M.; GRAY, K. The Neuropsychiatric Inventory: comprehensive assessment of psychopathology in dementia. Neurology, St. Paul, v. 44, p. 2308-2314,1994.

CUMMINGS, J. Toward a molecular neuropsychiatry of neurodegenerative diseases. Annals_of_Neurology, Malden, v. 54, p. 147-154, 2003.

HAMILTON, M. A rating scale for depression. Journal of Neurology, Neurosurgery \& Psychiatry, London, v. 23, p. 56-62, 1960.

HARWOOD, D.G.; BARKER, W.W.; OWNBY, R. L.; DUARA, R. Relationship of behavioral and psychological symptoms to cognitive impairment and functional status in Alzheimer's disease. International Journal of Geriatric Psychiatry, Malden, v. 15, p. 393-400, 2000.

HERNANDEZ, S. S. S.; COELHO, F. G. M.; GOBBI, S.; STELLA, F. Effects of physical activity on cognitive functions, balance and risk of fall in elderly with Alzheimer's dementia. Revista Brasileira de Fisioterapia, São Carlos, v. 14, n. 1, p. 68-74, 2010.
HEYN, P. The effect of a multisensory exercise program on engagement, behavior, and selected physiological indexes in persons with dementia.

American Journal of Alzheimers Disorder and Other Dementias, Thousand Oaks, v. 1, n. 4, p. 247-251, 2003.

HEYN, P.; ABREU, B. C.; OTTENBACHER. K. $J$. The Effects of Exercise Training on Elderly Persons With Cognitive Impairment and Dementia: A Meta-Analysis. Archives of Physical Medicine and Rehabilitation, Reston, v. 85, p. 1694-1704, 2004.

LYKETSOS, C. G.; SHEPPARD, J. M.; STEINBERG, M. Neuropsychiatric disturbance in Alzheimer's disease clusters into three groups: the Cache County study. International Journal of Geriatric Psychiatry, Malden, v. 16, p. 10431053, 2001.

MAGNI, R.; BINETTI, G.; BIANCHETTI, A.; TRABUCCHI, M. Risk of mortality and institutionalization in demented patients with delusion. Journal of Geriatric Psychiatry and Neurology, Thousand Oaks, v. 9, p. 123-126, 1996.

MCCURRY, S. M.; LOGSDON, R. G.; TERI, L. et al. Characteristics of sleep disturbance in communitydwelling Alzheimer's disease patients. Journal of Geriatric Psychiatry and Neurology, Thousand Oaks, v. 12, p. 53-59, 1999.

MCCURRY, S. M.; REYNOLDS, C. F.; ANCOLIISRAEL, S.; LINDA, T.; VITIELLO, M. V.

Treatment of sleep disturbance in Alzheimer's disease. Sleep Medicine Reviews, Atlanta, v. 4, n. 6, p. 603-628, 2000.

MCCURRY, S. M.; LOGSDON, R. G.; VITIELLO, M. V.; TERI, L. Treatment of sleep and nighttime disturbances in Alzheimer's disease: a behavior management approach. Sleep Medicine, Atlanta, v. 5 , p. $373-377,2004$.

MCCURRY, S. M.; GIBBONS, L. E.; LOGSDON, R. G.; VITIELLO, M. V.; TERI, L. Nighttime Insomnia Treatment and Education for Alzheimer's Disease: a Randomized, Controlled Trial. Journal of the American Geriatrics Society, Los Angeles, v. 53, p. 793-802, 2005

MCKHANN, G.; DRACHMAN, D.; FOLSTEIN, M. Clinical Diagnosis of Alzheimer's Disease. Reporto of the NINCD-ADRA Work Group under the anspices of Department of Health and Human Services Task Force on Alzheimer's Disease. Neurology. St. Paul, v. 34, p. 939-944, 1984.

MEGA, M.; CUMMINGS, J.L.; FIORELLO, T.; et al. The spectrum of behavioral changes in 
Alzheimer's disease. Neurology, St. Paul, v. 46, p. 130-135, 1996.

MILLER, R. J.; SNOWDON, J.; VAUGHAN. R. The use of the Cohen-Mansfield Agitation Inventory in the assessment of behavioral disorders in nursing homes. Journal of Americam Geriatric Society, Los Angeles, v. 43, p. 546-549, 1995.

MORRIS, J. C.; HEYMAN, A.; MOHS, R. C.; HUGHES, M. S.; VAN BELLE, G.; FILLENBAUM, G.; MELLITS, E. D.; CLARK, C. The Consortium to Establish a Registry for Alzheimer's Disease (CERAD). Part I. clinical and neuropsychological assessment of Alzheimer's disease. Neurology, St. Paul, v. 39, p. 1159-1165, 1989.

MULLER-THOMSEN, T.; ARLT, S.; MANN, U. Detecting depression in Alzheimer's disease: Evaluation of four different scales. Archives of Clinical Neuropsychology, Atlanta, v. 20, p. 271276, 2005.

NAMAZI, K. H.; PAULLETTA, B. G.; ZADOROZNY, C. A. A low intensity exercise/movement program for patients with Alzheimer's Disease: The TEMP-AD Protocol. Journal of Aging and Physical Activity, Champaign, v. 2, p. 80-92, 1994.

NAMAZI, K. H.; ZADOROZNY, C. A.; PAULLETTA, B. G. The Influences of Physical Activity on Patterns of Sleep Behavior of Patients with Alzheimer's disease. International Journal of Aging and Human Development, Amityville, v. 40 , p. $145-153,1995$.

NITRINI, R.; CARAMELLI, P.; HERRERA, E. J. Performance of illiterate and literate nondemented elderly subjects two tests of long-term memory. Journal of the International Neuropsycholical Society. Albuquerque, v. 10, n. 4, p. 634-638, 2004.

ONOR, M. L.; TREVISIOL, M.; NEGRO, C.; AGUGLIA, E. Different perception of cognitive impaiment, behavioral disturbances, and functional disabilities between persons with mild cognitive impaiment and mild Alzheimer's disease and their caregivers. American Journal of Alzheimer's Disease and other Dementias. Thousand Oaks, v. 5, n. 21, p. 333-338, 2006.

\section{ORGANIZAÇÃO MUNDIAL DA SAÚDE. Manual de classificação internacional de doenças, lesões e causas de óbitos. 9. rev. 1975. São Paulo: Centro da OMS para Classificação de Doenças em Português, 1978.}

PLATI, M. C. F.; COVRE, P.; LUKASOVA, K.; MACEDO, E. C. Depressive symptoms and cognitive performance of the elderly: relationship between institutionalization and activity programs. Revista Brasileira de Psiquiatria, São Paulo, v. 28, n. 2, p. 118-221, 2006.

RITCHIE, K. Behavioral disturbances of dementia in ambulatory care settings. International psychogeriatric, Victoria, v. 8, n.3, p. 439-442, 1996.

ROLLAND, Y.; PILLARD, F.; KLAPOUSZCZAK, A.; REYNISH, E.; THOMAS, D.; ANDRIEU, S.; RIVIÈRE, D.; VELLAS, B. Exercise Program for Nursing Home Residents with Alzheimer's disease: A 1-Year Randomized, Controlled Trial. Journal of American Geriatrics Society. Los Angeles, v. 55, p. 158-165, 2007.

ROSEN, J.; BURGIO, L.; KOLLAR, M. The Pittisburg Agitation Scale: a user-friendly instrument for rating agitation in dementia patients. Journal of American Geriatric Psychiatry, Bethesda, v. 2, 1991.

SASAKI, M.; ARAI, Y.; KUMMAMOTO, K.; ABE, K.; ARAI, A.; MIZUNO, Y. Factors related to potentially harmful behaviors towards disable older people by family caregivers in Japan.

International Journal of Social Psychiatry, Thousand Oaks, v. 22, p. 250-257, 2007.

SCARMEAS, N.; BRANDT, J.; ALBERT, M.; HADJIGEORGIOU, G.; PAPADIMITRIOU A DUBOIS, B. Delusions and hallucinations are associated with worse outcome in Alzheimer disease. Archives of Neurology, Chicago, v. 62, p. 1601-1608, 2005.

SEOW, D.; GAUTHIER, S. Pharmacotherapy of Alzheimer disease. Canadian Journal of Psychiatry. Ottawa, v. 52, n. 10, p. 620-229, 2007.

STEINBERG, M.; LEOUTSAKOS, J. M. S.; PODEWILS, L. J.; LYKETOS, C. G. Evaluation of a home-based exercise program in the treatment of Alzheimer's disease: the maximizing independence in dementia (MIND) study. International Journal of Geriatric Psychiatry, Malden, v. 24, n. 7, p. 680-685, 2008.

TAN, L. L.; WONG, H. B.; ALLEN, H. The impacto $f$ neuropsychiatric symptoms of dementia on distress in family and Professional caregivers in Singapore. International Psychogeriatric, Victoria, v. 17, p. 253-263, 2005.

TATSCH, M. F.; BOTTINO, C. M. C.; AZEVEDO, D.; HOTOTIAN, S. R.; MOSCOSO, M. A.; FOLQUITTO, J. C.; SCALCO, A. Z.; LOUZÃ, M. R. Neuropsychiatric Symptoms in Alzheimer Disease and Cognitively Impaired, Nondemented Elderly From a Community-Based Sample in Brazil: Prevalence and Relationship With 
Dementia Severity. American Journal of

Geriatric Psychiatry. Bethesda, v. 14, n. 5, p.

438-445, 2006.

TERI, L.; LARSON, E. B.; REIFLER, B. V. Behavioral disturbance in dementia of the Alzheimer's type. Journal of American Geriatric Society, Los Angeles, v.36, n.1, p. 1-6, 1988.

TERI, L.; GIBBONS, L. E.; MCCURRY, S.; LOGSDON, R.; BUCHNER, D. M.; BARLOW, W. E.; KUKULL, W. A.; LACROIX, A. Z.; MCCORMICK, W.; LARSON, E. B. Journal of American Medical Association, Chicago, v.209, n. 15, p. 2015-2022, 2003.

TUN, S. M.; MURMAN, D. L.; LONG, H. L.; COLENDA, C. C.; VON EYE, A. Predictive validity of neuropsychiatric subgroups on nursing home plecement and survival in patients with Alzheimer disease. American Journal of Geriatric

Psychiatry, Bethesda, v. 15, p. 314-332, 2007.

VANCE, D. E.; WADLEY, V. G.; BALL, K. K.; ROENKER, D. L.; RIZZO, M. The effects of physical activity and sedentary behavior on cognitive health in older people. Journal of Aging Physical Activity, Champaign, v. 13, p. 294-313, 2002.

YESAVAGE, J.; BRINK, T.; ROSE, T.; LUM, O.; HUANG, V.; ADEY, M.; LEIRER, V. Development and validation of a geriatric depression screenning scale: a preliminary report. Journal of Psychiatry Research, Atlanta, v. 17, p. 37-49, 1983.

Agradecimento: LAFE; FINEP; FNS-MS; FUNDUNESP; PROEX-UNESP; UNESP-UNATI; CNPQ.

Endereço:

Salma Stéphany Soleman Hernandez

Av. 3A, 931/apto 01 Cidade Nova

Rio Claro SP Brasil

13506-790

Telefone: (19) 93450827

e-mail: salma_soleman@yahoo.com.br

Recebido em: 17 de fevereiro de 2010.

Aceito em: 15 de março de 2011.

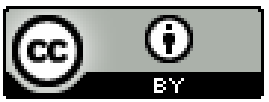

Motriz. Revista de Educação Física. UNESP, Rio Claro, SP, Brasil - elSSN: 1980-6574 - está licenciada sob Creative Commons - Atribuição 3.0 